\title{
How many histological levels should be examined from tissue blocks originating in cone biopsy and large loop excision of the transformation zone specimens of cervix?
}

\author{
M K Heatley
}

\begin{abstract}
Aims-To establish the value of examining additional histological levels in cone biopsy and large loop excision of the transformation zone (LLETZ) specimens of cervix.

Methods-Three deeper levels were examined from 200 consecutive cone biopsy and LLETZ specimens reported by a single pathologist.

Results-Examination of the first deeper level resulted in cervical intraepithelial neoplasia (CIN) being identified for the first time in five cases and in CIN1 being upgraded in five more. Invasive cancer was discovered in two cases that had shown high grade CIN initially.

Conclusion-Examination of a single further level appears to be sufficient in those patients in whom a specimen is compromised because epithelium including the squamocolumnar junction is missing, or if there is a discrepancy between the histological findings and the preceding colposcopic or cytological history. If invasive disease is suspected on the basis of the cytological, colposcopic, or histological features, one or preferably two further levels should be examined. (F Clin Pathol 2001;54:650-651)
\end{abstract}

Keywords: cervix uteri; quality control; diagnosis

A recent working party of the Royal College of Pathologists and the NHS Cervical Screening Programme recommends that examination of a single level from each block from a cone biopsy or large loop excision of the transformation zone (LLETZ) of the cervix may suffice, but that further levels are mandatory if there are difficulties in identifying the source of abnormal cells seen in a preceding cervical smear, or if there are features associated with an increased risk of invasive disease. ${ }^{1}$ This reflects the policy described in a standard textbook, ${ }^{2}$ but neither document indicates the number of levels that should be examined, although examination of three histological levels from the blocks originating in punch biopsies of the cervix is advocated. ${ }^{2}$ The purpose of this study was to determine how many histological levels should be examined from blocks that originated in a cervical cone biopsy or LLETZ.

Materials and methods

In our department, cone biopsies and LLETZ specimens are cut in parallel slices at $3 \mathrm{~mm}$ intervals using a $3 \mathrm{~mm}$ plastic tissue slicer (Medim Histotechnology, South Godstone, Surrey, UK), with each piece of tissue being embedded in an individual cassette. A single haematoxylin and eosin section is examined initially with a further three sections, four in all, being examined if the tissue is incomplete, if there is no evidence of cervical intraepithelial neoplasia (CIN), if there are features that are suspicious of invasive disease, ${ }^{3}$ or if there is a discrepancy between the clinical, colposcopic, or cytological findings and the features present on histological examination of the sections from the cone biopsy or LLETZ.

The findings in 200 consecutive cone biopsy and LLETZ specimens reported by a single gynaecological pathologist in which additional levels had been examined were studied prospectively. Cases in which there was no discrepancy between clinical, colposcopic, and histological findings, in which there was no suspicion of invasive disease, in which the CIN lesion was clear of the specimen edges, and in which a complete initial section was obtained were excluded. The highest grades of CIN, if present, in the initial, first deeper level, second deeper level, and third deeper level were compared for all 200 cases. Comparisons were also made for the following subgroups: cases in which the epithelium, including that at the squamocolumnar junction, was missing or in which there was extensive diathermy artefact; cases in which invasive disease was suspected histologically or on the basis of the reported cytological or colposcopic findings, and cases in which there was no evidence of a CIN lesion on the initial level or in which there was more than one grade of difference between the histological diagnosis and preceding cytological, clinical, or colposcopic features.

\section{Results}

Of the 200 specimens, 170 were intact, 21 were incomplete, usually because one or both lateral edges were missing, and nine were received in two or more fragments. Table 1 includes details of the maximum basal dimension, apical height, and number of blocks from each group. Fragmented specimens tended to be shallower and to yield a greater number of blocks than either intact or incomplete samples. The condition of the specimen was not associated with a change in histological grade when deeper levels were examined. Table 2 describes the findings on the initial level and the first level for the cases reviewed. Examination of the first 
Table 1 Details of the condition in which the specimens were received and the association with specimen size, number of blocks, and change in grade upon examining deeper levels histologically

\begin{tabular}{|c|c|c|c|c|c|}
\hline & Intact & Incomplete & & Fragmented & Total \\
\hline Number of specimens & 170 & 21 & & 9 & 200 \\
\hline \multicolumn{6}{|c|}{ Maximum basal dimension (mm) } \\
\hline Mean & 21.0 & 22.9 & & 23.9 & 21.3 \\
\hline Minimum & 7.0 & 12.0 & & 15.0 & 7.0 \\
\hline \multirow{2}{*}{ Maximum } & 40.0 & 40.0 & & 32.0 & 40.0 \\
\hline & & & $\mathrm{p}=0.269$ & & \\
\hline \multicolumn{6}{|c|}{ Maximum apical height (mm) } \\
\hline Mean & 13.6 & 12.9 & & 7.9 & 13.25 \\
\hline Minimum & 2.0 & 4.0 & & 2.0 & 2.0 \\
\hline \multirow[t]{2}{*}{ Maximum } & 35.0 & 26.0 & & 13.0 & 35.0 \\
\hline \multirow{2}{*}{\multicolumn{6}{|c|}{ Number of blocks }} \\
\hline & & & & & \\
\hline Mean & 7.6 & 8.6 & & 11.8 & 7.85 \\
\hline Minimum & 2.0 & 4.0 & & 4.0 & 2.0 \\
\hline \multirow[t]{2}{*}{ Maximum } & 13.0 & 14.0 & & 23.0 & 23.0 \\
\hline & & & $\mathrm{p}=0.003$ & & \\
\hline \multicolumn{6}{|l|}{ Change in grade } \\
\hline Yes & 10 & 1 & & 1 & 12 \\
\hline No & 160 & 21 & & 8 & 188 \\
\hline
\end{tabular}

The dimensions and number of blocks were compared statistically with the condition of the specimen using the Kruskal-Walls test.

The change in grade was compared with the condition of the specimen using the $\chi^{2}$ test.

Table 2 The highest grade of CIN in the initial histological section and the first (deeper) level in 200 cervical cone biopsies and LLETZ specimens in which the blocks were examined through multiple histological levels

\begin{tabular}{|c|c|c|c|c|c|}
\hline & & \multicolumn{4}{|c|}{ First deeper level } \\
\hline & & Negative & Low grade & High grade & Invasive \\
\hline \multicolumn{6}{|l|}{ All cases } \\
\hline \multirow[t]{4}{*}{ Initial section } & Negative & 26 & 4 & 1 & 0 \\
\hline & Low grade & 0 & 37 & 5 & 0 \\
\hline & High grade & 0 & 1 & 113 & 2 \\
\hline & Invasive & 0 & 0 & 0 & 11 \\
\hline \multicolumn{6}{|c|}{ Squamocolumnar junction missing, cautery artefact, or no epithelium } \\
\hline \multirow{3}{*}{ Initial section } & Negative & 3 & 0 & 1 & 0 \\
\hline & Low grade & 0 & 22 & 2 & 0 \\
\hline & High grade & 0 & 1 & 64 & 0 \\
\hline \multicolumn{6}{|c|}{ Possible invasive disease based on histological features or history of cytological or colposcopic suspicion } \\
\hline \multirow[t]{4}{*}{ Initial section } & Negative & 4 & 1 & 0 & 0 \\
\hline & Low grade & 0 & 4 & 1 & 0 \\
\hline & High grade & 0 & 0 & 47 & 2 \\
\hline & Invasive & 0 & 0 & 0 & 11 \\
\hline \multicolumn{6}{|c|}{$\begin{array}{l}\text { No cin on initial section or more than } 1 \text { grade difference between histological appearances and cytologica } \\
\text { or colposcopic appearances }\end{array}$} \\
\hline \multirow[t]{3}{*}{ Initial section } & Negative & 19 & 3 & 0 & 0 \\
\hline & Low grade & 0 & 9 & 4 & 0 \\
\hline & High grade & 0 & 0 & 1 & 0 \\
\hline
\end{tabular}

The data are also presented for those subgroups in which there was trauma to the sample so that some epithelium was not represented in the initial section, where there was a suggestion that invasive disease may be present, and where a higher grade of CIN could be present.

CIN, cervical intraepithelial neoplasia; LLETZ, large loop excision of the transformation zone.

deeper level resulted in $12(6 \%)$ of the cases being upgraded. In five cases where there had been no evidence of CIN on the initial section, low grade or high grade disease was identified, and in a further five low grade cases, high grade disease was identified on this first deeper level. In two instances, the presence of invasive disease was discovered after examination of the additional first deeper level from specimens showing a high grade CIN lesion. In one instance, examination of an additional level showed that the high grade lesion had cut out and that low grade disease was present deeper in the blocks.

Cutting of additional sections because part of the transformation zone, including the squamocolumnar junction, was missing or because epithelium had been stripped from the surface of the section (possibly owing to diathermy artefact) did not yield complete sections with an intact epithelium in an appreciable number of cases, but it did result in the identification of a high grade lesion in one case, which had previously been regarded as negative, and in two cases where only a low grade lesion had been identified previously. Suspicion of invasive disease based on histological findings in the specimen, or because of a history of cytological abnormality or colposcopic suspicion of invasion, allowed invasive disease to be identified in two cases. In one further case, invasive disease that had not been present in the initial or first deeper level was identified on a second deeper level. Cutting a third deeper level did not appear to improve the yield of invasive disease in these cases, although on occasion, the profile occupied by the invasive focus increased in size. A higher grade of CIN was identified in seven of the 36 women in whom there was no evidence of CIN in the initial section from the cone biopsy or LLETZ specimen and those in whom there was a discrepancy between the findings in the definitive and original samples.

\section{Discussion}

The findings re-enforce the advisability of selectively examining further material in cone biopsy and LLETZ specimens from the cervix, as recommended in standard texts. ${ }^{12}$ Examination of a single further level appears to suffice in those individuals in whom the specimen is compromised because the squamocolumnar junction or other epithelium is missing when there is no evidence of CIN or a CIN lesion is confined to a low grade lesion. Within this subgroup, invasive foci were not identified in any of the cases in which high grade disease had been identified on the initial histological level. A similar practice is advisable if there is no evidence of a CIN lesion on the initial section.

A suspicion that invasive disease may be present based on the histological features of the material examined previously, or on the basis of a clinical, colposcopic, or cytological suspicion of invasive disease would appear to justify a further two levels. Obviously, if further suspicious areas are identified, the pathologist may elect to examine additional material. Although it has been suggested that, from a purely pragmatic point of view, an area of invasion too small to be identified on sections taken at 2-3 $\mathrm{mm}$ intervals is too small to result in any change in the management of the patient, ${ }^{2}$ this study provides evidence to support a practical policy that combines patient safety and appropriate management of scarce laboratory resources.

I am grateful to Miss K Newby for typing the manuscript.

1 Fox H. Working party of the Royal College of Pathologists and the NHS Cervical Screening Programme. Histopathology reporting in cervical screening. NHSCSP Publication No 10. NHSCSP, 1999:45.

2 Anderson MC. Invasive carcinoma of the cervix. In: Anderson MC, ed. Female reproductive system. Systemic pathology. Edinburgh: Churchill Livingstone, 1991:122-3.

3 Al Nafussi AI, Hughes DE. Histological features of CIN3 and their value in predicting invasive microinvasive squamous carcinoma. f Clin Pathol 1994;47:799-804. 